\title{
Directed Hypersonic Strain Waves Imaged with Ultrafast Electron Microscopy
}

\author{
Daniel X. Du ${ }^{1}$, Daniel R. Cremons ${ }^{1}$ and David J. Flannigan ${ }^{1}$
}

${ }^{1}$. Department of Chemical Engineering and Materials Science, University of Minnesota, Minneapolis, MN.

Intense, ultrafast photoexcitation of semiconducting materials produces several discrete but inter-related energy relaxation processes that span large spatial and temporal scales [1]. Initial charge-carrier excitation, and generation of a dense electron-hole plasma, produces hypersonic plasma waves that propagate outward from the excitation zone. Related to this, myriad scattering processes (electronelectron, electron-phonon, etc.) occur within the first few picoseconds following photoexcitation, ultimately leading to the launch of coherent propagating strain waves [2]. In thin membranes of semiconducting materials, propagating strain waves display Lamb-type symmetries and hypersonic velocities (depending on mode type and membrane thickness) [3]. In addition to often-used ultrafast spectroscopic methods, femtosecond X-ray and electron scattering has been used to study both hypersonic plasma waves and acoustic-phonon wavetrains [4,5]. Observations from these experiments, combined with knowledge of the associated spatiotemporal scales, lead to questions about preferred nucleation points/regions, propagation directions, and time-varying phase-velocity dispersion behaviors.

Here, we discuss the results of real-space imaging, with ultrafast electron microscopy (UEM) [6], of hypersonic coherent strain-wave dynamics in Ge [7]. While much is known about ultrafast chargecarrier dynamics in semiconductors like Ge and GaAs, several open questions remain pertaining to local acoustic-phonon behaviors and possible connections to hypersonic plasma waves [8]. Specimens consisted of $2^{\circ}$ wedge-polished Ge single crystals. The polishing process employed produces linear nanoscale striations on the polished surface having a repeating ridge-like morphology [Figure 1(a,b)]. Intense, in situ femtosecond photoexcitation of these specimens produces coherent acoustic-phonon wavetrains that propagate parallel to the striations and with initial phase velocities well in excess of the bulk speed of sound [e.g., $35 \mathrm{~km} / \mathrm{s}$ vs. $5 \mathrm{~km} / \mathrm{s}$, respectively; Figure 1(c,d)]. Further, while the phase velocity of each wavefront is fixed across the micrometer imaging field of view, each subsequent wavefront has a slightly reduced velocity, ultimately decaying to the approximate bulk speed of sound over one nanosecond [Figure 1(e)]. Comparison of this imaged time-varying phase-velocity dispersion behavior to calculated guided acoustic modes suggests a single, first-order symmetric Lamb mode is responsible for the observed contrast dynamics [Figure 1(f)]. By using a plasma lensing method to determine time zero (moment of in situ specimen photoexcitation) [9], a significant delay (100 ps) was observed to occur before emergence of the first coherent contrast wave (a total of 200 ps passed before quantifiable waves were observed). This suggests a connection between Auger recombination dynamics and strain-wave nucleation and launch [10].

References:

[1] A. Othonos, J. Appl. Phys. 83 (1998), p. 1789.

[2] P. Ruello, V. E. Gusev, Ultrasonics 56 (2015), p. 21.

[3] J. Cuffe et al, Nano Lett. 12 (2012), p. 3569.

[4] M. F. DeCamp et al, Phys. Rev. Lett. 91 (2003), p. 165502.

[5] M. Harb et al, Phys. Rev. Lett. 100 (2008), p. 155504. 
[6] D. A. Plemmons, P. K. Suri, D. J. Flannigan, Chem. Mater. 27 (2015), p. 3178.

[7] D. R. Cremons, D. X. Du, D. J. Flannigan, Phys. Rev. Mater. 1 (2017), p. 073801.

[8] B. Y.-K. Hu, C. J. Stanton, J. W. Wilkins, Phys. Rev. B 44 (1991), p. 11067.

[9] D. A. Plemmons, D. J. Flannigan, Chem Phys. Lett. 683 (2017), p. 186.

[10] This material is based on work supported by the U.S. Department of Energy, Office of Science, Office of Basic Energy Sciences under Award No. DE-SC-0018204. This work was supported partially by the National Science Foundation through the University of Minnesota MRSEC under Award No.

DMR-1420013. This work was also partially supported by the Arnold and Mabel Beckman Foundation through a 2015 Beckman Young Investigator Award. We thank Stefano Gonella for discussions pertaining to complex guided-wave phenomena and Andre Mkhoyan for use of his wedge polishing system.
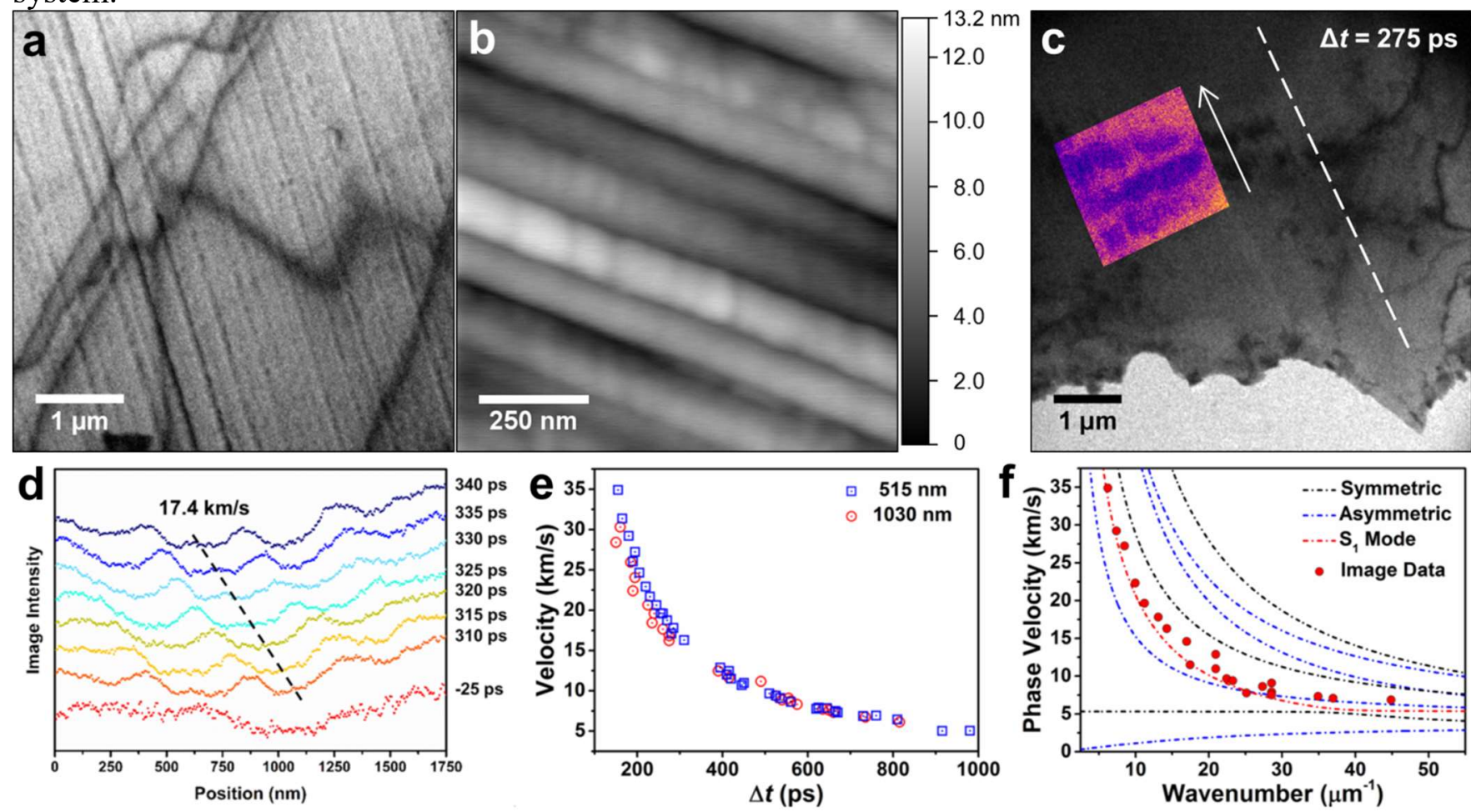

Figure 1. Directed hypersonic acoustic-phonon wavefronts in Ge. (a) Bright-field TEM image of linear polishing striations in a $2^{\circ} \mathrm{Ge}$ wedge specimen. (b) AFM image (tapping mode) of the polished Ge surface (average ridge width $=110 \pm 30 \mathrm{~nm} ; n=19$ ridges; error is one standard deviation). (c) Brightfield UEM image obtained 275 ps after in situ femtosecond photoexcitation $(\Delta t)$. The false-colored region is meant to highlight contrast features arising from a coherent wavetrain, and the arrow indicates the wavefront propagation direction. The dashed line marks the general linear striation orientation. (d) Line scans from select UEM images (e.g., 310 ps, 315 ps, 320 ps, etc.) from the false-colored region shown in (c). One wavefront propagating at $17.4 \mathrm{~km} / \mathrm{s}$ is highlighted with a dashed black line. A preexcitation line scan (-25 ps) is shown for comparison. (e) Time-varying phase-velocity dispersion of propagating acoustic phonons generated with two different photoexcitation wavelengths (515 and 1,030 $\mathrm{nm}$, blue squares and red circles, respectively). Time zero was determined with a plasma lensing method [9], and the first quantifiable wavefronts occurred 200 ps after photoexcitation. (f) Experimental phase-velocity dispersion (red dots) compared to calculated guided acoustic modes (dot-dashed lines). Symmetric modes are shown in black, and asymmetric modes are shown in blue. The first-order symmetric mode is shown in red and most closely matches the experiment. 